\title{
An approach to neck masses in adults
}

Eren Tan, Joseph Jaya

\section{Background \\ Neck masses in adults are a common presentation for head and neck cancer. Head and neck cancer accounts for 3.4\% of all malignancies in Australia, and the incidence of oropharyngeal squamous cell carcinoma is rising. Early diagnosis is essential to prevent worsening prognosis.}

\section{Objective}

This article provides a brief overview of neck masses in adults, with a guideline to work-up and management in a primary care setting.

\section{Discussion}

All neck masses should be considered malignant until proven otherwise. Detailed history and examination is crucial in the initial work-up. Fine-needle aspiration and computed tomography of the neck with contrast make up the mainstay of first-line investigation.
NECK MASSES are a common cause of presentation to general practitioners (GPs) and may be the only presenting complaint of a patient with head and neck malignancy. ${ }^{1}$ In adults, head and neck malignancy is the most common cause for neck masses, ${ }^{2,3}$ and it accounts for $3.4 \%$ of all malignancies in Australia. ${ }^{4}$ Delays in diagnosis of up to 180 days are not uncommon, ${ }^{5}$ with referral delay being associated with a three-fold increase in mortality. ${ }^{5}$ Given the incidence of oropharyngeal squamous cell carcinoma (SCC) in Australia is rising, ${ }^{6-8}$ this should prompt the need for increased vigilance; early referral to a specialist ear, nose and throat (ENT) service is prudent if any concerns or uncertainty in management is encountered. For these reasons, all adult neck masses should be considered malignant until proven otherwise. ${ }^{9}$

The purpose of this article is to provide a systematic approach to the work-up of neck masses, including pertinent signs and symptoms, recommended investigations and when to refer for ENT specialist management.

\section{Human papillomavirus}

In recent years, the rates of human papillomavirus (HPV)-positive oropharyngeal cancer have risen markedly, with the prevalence more than tripling from $19 \%$ to $66 \%$ between 1987 and 2006..$^{10,11}$ The overall rate of oropharyngeal cancer is increasing despite lower rates of tobacco use. ${ }^{6-8}$

When compared with HPV-negative oropharyngeal malignancy, patients with HPV-positive head and neck cancer often do not fit the stereotype for a patient with malignancy (Box 1), which results in a delayed cancer diagnosis. ${ }^{12}$

HPV-positive head and neck cancers are more likely to present with an asymptomatic neck mass, ${ }^{13}$ and the neck nodes are frequently cystic. These are often misdiagnosed as branchial cleft cysts, ${ }^{14}$ leading to delayed diagnosis and delayed treatment. Given the rise of HPV-related head and neck cancer, the possibility of an incorrect diagnosis must always be considered.

\section{Initial assessment}

Thorough history-taking is vital, and the presence of any red flag symptoms or risk factors should be documented.

Red flag symptoms for head and neck cancer include: ${ }^{9}$

- a mass that has been present for $>2$ weeks

- a recent voice change

- dysphagia or odynophagia

- ipsilateral otalgia, nasal obstruction or epistaxis

- unexplained weight loss or loss of appetite. Risk factors for head and neck cancer include: ${ }^{9,15}$

- smoking

- alcohol use

- age $>40$ years

- past history of previous head and neck malignancy

- past history of head and neck cutaneous lesions.

Assessing the location of a neck mass is essential as it provides clues to the aetiology. Emphasis should be placed on documenting the location accurately. The neck is divided into six levels, each 
describing a lymph node region, with the midline being the central dividing line between left and right (Figure 1): $:^{16,17}$

- Level I: the submental and submandibular triangles

- Level II, III, IV: the upper, middle and lower internal jugular chain

- Level V: the posterior triangle

- Level VI: the anterior compartment. Further assessment should pay specific attention to characterising other features of the neck mass and completing a full head and neck examination.

Other features of the neck mass that require characterisation are: ${ }^{18}$

- mobility of the neck mass (fixed masses are more likely to be malignant)

- size ( $>1.5 \mathrm{~cm}$ is more likely to be malignant)

- firmness

- overlying skin ulceration.

Examination of the head and neck includes:

- inspection for cutaneous lesions

- otoscopy (unilateral middle ear effusion may indicate nasopharyngeal carcinoma)

- anterior rhinoscopy

- oral cavity inspection and palpation

- oropharyngeal inspection (looking for masses, ulceration)

- tonsil enlargement or asymmetry

- flexible nasopharyngolaryngoscopy.

\section{Investigations}

First-line investigations for all adults at risk of malignancy consist of computed tomography (CT) of the neck with contrast and fine-needle aspiration (FNA). These two investigations provide complementary information including primary tumour histopathological detection, anatomical localisation and nodal staging. Simultaneous arrangement of these investigations prevents delays in diagnosis and treatment.

\section{Computed tomography of the neck with contrast}

Contrast-enhanced CT of the neck should be considered a first-line investigation for all patients at increased risk of malignancy. ${ }^{19}$ This will assist in the localisation of the primary neoplasm, assess for further cervical lymphadenopathy and aid in the staging process. Furthermore, it may provide information that suggests a benign process rather than malignancy (eg salivary calculi, dental infection). Contrast should always be administered for enhanced soft-tissue differentiation unless a prior contraindication is present (eg iodine allergy, renal impairment). ${ }^{19}$ Although contrast-enhanced CT of the neck is considered a first-line investigation, studies have shown a negative predictive value of 0.33 for the detection of primary tumours

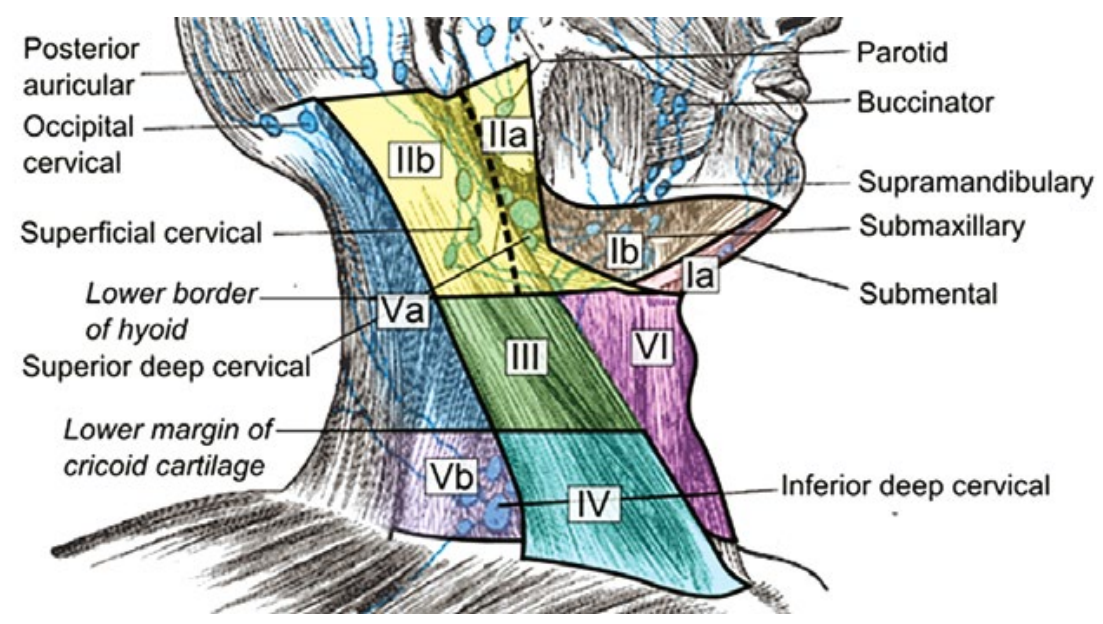

Figure 1. Anatomical diagram depicting the six levels of the neck

and 0.57 for the detection of recurrent tumours in the head and neck. ${ }^{20}$ For this reason, patients with a suspicious neck mass and normal contrast-enhanced CT of the neck should always undergo further evaluation by an ENT specialist.

\section{Fine-needle aspiration}

FNA should be considered a first-line diagnostic test for all patients at increased risk of malignancy. It is safe and cost effective and has an overall accuracy of $93.1 \%$ (73.3-98.0\%) according to a meta-analysis. ${ }^{21}$

In indeterminate cases, a repeat FNA or core biopsy may be performed. A meta-analysis showed that ultrasoundguided core biopsy was highly accurate (96\% detection rate for malignancy) with low complication rates (1\%). ${ }^{22}$

\section{Other investigations}

\section{Ultrasonography}

Diagnostic ultrasonography is not recommended as a first-line investigation in preference to cross-sectional imaging (eg contrast-enhanced CT or magnetic resonance imaging [MRI]) as it is highly operator dependent. While it is useful to assist in obtaining an image-guided aspiration sample, the lack of visualisation of deeper structures limits its usefulness as an investigation.

\section{Magnetic resonance imaging of the neck with contrast}

Although MRI provides better soft-tissue contrast, ${ }^{19} \mathrm{CT}$ is the preferred primary imaging modality, as MRI demonstrates

\section{Box 1. Features suggestive of human papillomavirus-positive oropharyngeal cancer ${ }^{24,25}$}

Younger age

Male sex

Higher number of oral and vaginal sexual partners

Less or no tobacco exposure

Less alcohol consumption

Marijuana use

Higher education

Higher socioeconomic status 
more motion artefact, longer scan times and generally poorer availability and tolerability.

\section{Positron emission tomography with computed tomography}

Positron emission tomography (PET) with CT using fluorodeoxyglucose tracer has shown excellent sensitivity and specificity in the assessment of primary and recurrent head and neck tumours. ${ }^{20}$ As such, PET/ $\mathrm{CT}$ is playing an increasingly greater part in the work-up of head and neck tumours, especially in the detection of residual or recurrent tumours post-treatment. ${ }^{23}$ However, given its limited availability, PET/CT is not appropriate as a first-line imaging study.

\section{Ancillary investigations}

Ancillary tests are intended to assist clinicians when malignancy is unlikely or when initial investigations do not yield a diagnosis. If these tests are undertaken, they should be based on clinical suspicion for specific diseases and should be obtained simultaneously to the malignancy work-up to prevent a delayed diagnosis. Ancillary investigations are shown in Table $1 .{ }^{9}$

\section{Investigation process}

Investigation of neck masses relies on excluding malignancy. Deciding on the course of investigation depends on an assessment of signs and symptoms, and consideration of the results of previous investigations. At each stage of the investigative process, referral to a specialist ENT service should be considered to prevent referral delay. An approach to investigation is shown in Figure 2.

\section{Key points}

- Head and neck malignancy is the most common cause of adult neck masses.

- It is recommended that all adult neck masses be considered malignant until proven otherwise.

- All patients presenting with a neck mass should have a thorough history taken and examination performed followed by targeted investigations.
Table 1. Ancillary investigations for adult neck masses

\begin{tabular}{ll}
\hline Ancillary investigation & Rationale \\
\hline Full blood examination & $\begin{array}{l}\text { Elevated white cell count may indicate infection } \\
\text { or lymphoma }\end{array}$
\end{tabular}

Anti-neutrophil antibody (ANA)

Elevated ANA may indicate autoimmune diseases

Erythrocyte sedimentation rate (ESR) Elevated ESR may indicate autoimmune diseases

Thyroid stimulating hormone (TSH) TSH abnormalities may indicate thyroid pathology (eg multinodular goiter, Grave's disease)

Parathyroid hormone (PTH) Elevated PTH may indicate parathyroid adenoma

Thyroid ultrasonography May reveal thyroid nodules, parathyroid adenomas

Computed tomography of the chest with contrast

May reveal lung malignancy, tuberculosis or sarcoidosis

Specific infection tests (eg human

Positive tests may indicate infectious cause immunodeficiency virus, Epstein-Barr virus, cytomegalovirus, tuberculosis)

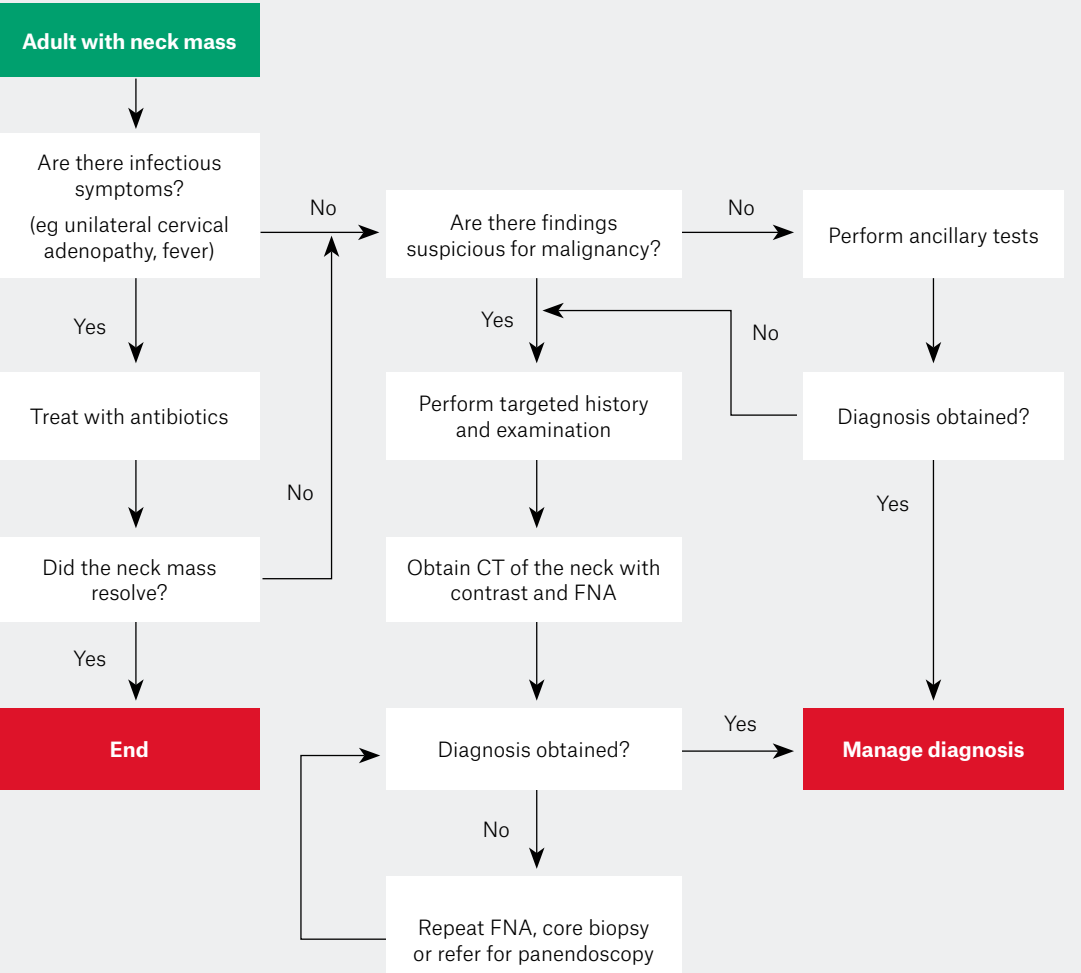

Figure 2. Flowchart for work-up of adults with a neck mass ${ }^{9}$ $C T$, computed tomography; FNA, fine-needle aspiration 
- It is important to continue investigations until a clear and specific diagnosis has been reached.

- CT of the neck with contrast and FNA are the mainstay of investigation for all patients with a neck mass suspicious for malignancy.

- Further evaluation by an ENT specialist is required for any patient with a suspicious neck mass and normal contrast-enhanced CT of the neck.

- Ancillary testing may be performed, without delaying investigation for malignancy.

- The advice of an ENT specialist can be sought if there are any concerns or uncertainties.

\section{Authors}

Eren Tan MBBS, Surgical Registrar, Eastern Health, Vic.eren.tan@live.com

Joseph Jaya MBBS, Surgical Registrar, Monash Health, Vic

Competing interests: None.

Funding: None.

Provenance and peer review: Not commissioned, externally peer reviewed.

\section{References}

1. Barakat M, Flood LM, Oswal VH, Ruckley RW. The management of a neck mass: Presenting feature of an asymptomatic head and neck primary malignancy? Ann R Coll Surg Engl 1987;69(4):181-84.

2. Beenken SW, Maddox WA, Urist MM. Workup of a patient with a mass in the neck. Adv Surg 1995;28:371-83.

3. Olsen KD. Evaluation of masses in the neck. Prim Care 1990;17(2):415-35

4. Australian Institute of Health and Welfare. Head and neck cancers in Australia. Cancer series no. 83. Cat. no. CAN 80. Canberra: AIHW, 2014.

5. Seoane J, Takkouche B, Varela-Centelles P, Tomás I, Seoane-Romero JM. Impact of delay in diagnosis on survival to head and neck carcinomas: A systematic review with metaanalysis. Clin Otolaryngol 2012;37(2):99-106 doi: 10.1111/j.1749-4486.2012.02464.x.

6. Chaturvedi AK, Anderson WF, Lortet-Tieulent J et al. Worldwide trends in incidence rates for oral cavity and oropharyngeal cancers. J Clin Oncol 2013:31(36):4550-59. doi: 10.1200/ JCO.2013.50.3870.

7. Hocking JS, Stein A, Conway EL, et al. Head and neck cancer in Australia between 1982 and 2005 show increasing incidence of potentially HPVassociated oropharyngeal cancers. Br J Cancer 2011;104(5):886-91. doi: 10.1038/sj.bjc.6606091.

8. Ariyawardana A, Johnson NW. Trends of lip, oral cavity and oropharyngeal cancers in Australia 1982-2008: Overall good news but with rising rates in the oropharynx. BMC Cancer 2013;13:333. doi: 10.1186/1471-2407-13-333.

9. Pynnonen MA, Gillespie MB, Roman B, et al. Clinical practice guideline: Evaluation of the neck mass in adults. Otolaryngol Head Neck Surg 2017;157(2_suppl):S1-30. doi: 10.1177/0194599817722550.

10. Hong A, Lee CS, Jones $D$, et al. Rising prevalence of human papillomavirus-related oropharyngeal cancer in Australia over the last 2 decades. Head Neck 2016;38(5):743-50. doi: 10.1002/hed.23942.

11. Hong $A M$, Grulich $A E$, Jones $D$, et al. Squamous cell carcinoma of the oropharynx in Australian males induced by human papillomavirus vaccine targets. Vaccine 2010;28(19):3269-72. doi: 10.1016/j.vaccine.2010.02.098.

12. Lewis A, Kang R, Levine A, Maghami E. The new face of head and neck cancer: The HPV epidemic. Oncol (Williston Park) 2015;29(9):616-26.

13. Carpén T, Sjöblom $A$, Lundberg $M$, et al. Presenting symptoms and clinical findings in HPVpositive and HPV-negative oropharyngeal cancer patients. Acta Otolaryngol 2018;138(5):513-18. doi: 10.1080/00016489.2017.1405279.

14. Wratten C, Anne S, Tieu MT, Kumar B, Eisenberg R. The dangers of diagnosing cystic neck masses as benign in the era of HPVassociated oropharyngeal cancer. Med J Aust 2015;203(9):371-72. doi: 10.5694/mja15.00697.

15. Singh J, Ramamoorthi R, Baxi S, Jayaraj R, Thomas M. The risk factors of head and neck cancer and their general patterns in Australia: $A$ descriptive review and update. J Environ Pathol Toxicol Oncol 2014;33(1):45-57. doi: 10.1615/ jenvironpatholtoxicoloncol.2014010130

16. Robbins KT, Shaha AR, Medina JE, et al. Consensus statement on the classification and terminology of neck dissection. Arch Otolaryngol Head Neck Surg 2008;134(5):536-38. doi: 10.1001/ archotol.134.5.536.

17. Amin MB, Edge S, Greene F, et al. AJCC cancer staging manual. Cham, Switzerland: Springer International Publishing, 2018.

18. Thakur JS, Sharma ML, Mohan C, Mohindroo NK, Kaushik NK. Clinicopathological and radiological evaluation of cervical lymph node metastasis in head and neck malignancies. Indian J Otolaryngol Head Neck Surg 2007;59(4):327-31. doi: 10.1007/ s12070-007-0094-2.

19. Aulino JM, Kirsch CFE, Burns J, et al. ACR Appropriateness Criteria ${ }^{\circledR}$ neck massadenopathy. J Am Coll Radiol 2019;16(5):S150-60. doi: 10.1016/j.jacr.2019.02.025.

20. Di Martino E, Nowak B, Hassan HA, et al. Diagnosis and staging of head and neck cancer: A comparison of modern imaging modalities (positron emission tomography, computed tomography, color-coded duplex sonography) with panendoscopic and histopathologic findings. Arch Otolaryngol Neck Surg 2000;126(12):1457-61. doi: 10.1001/archotol.126.12.1457.

21. Tandon S, Shahab R, Benton J, Ghosh SK, Sheard J, Jones T. Fine-needle aspiration cytology in a regional head and neck cancer center: Comparison with a systematic review and meta-analysis. Head Neck 2008;30(9):1246-52. doi: 10.1002/hed.20849.

22. Novoa E, Gürtler N, Arnoux A, Kraft M. Role of ultrasound-guided core-needle biopsy in the assessment of head and neck lesions: A metaanalysis and systematic review of the literature. Head Neck 2012;34(10):1497-503. doi: 10.1002/ hed.21821.

23. Fukui MB, Blodgett TM, Snyderman $\mathrm{CH}$, et al. Combined PET-CT in the head and neck: Part 2. Diagnostic uses and pitfalls of oncologic imaging. Radiographics 2005;25(4):913-30. doi: 10.1148/ rg. 254045136.
24. Gillison ML, D'Souza G, Westra W, et al. Distinct risk factor profiles for human papillomavirus type 16-positive and human papillomavirus type 16-negative head and neck cancers. J Natl Cancer Inst 2008;100(6):407-20. doi: 10.1093/jnci/djn025

25. Gillison ML, Castellsagué X, Chaturvedi A, et al. Eurogin roadmap: Comparative epidemiology of HPV infection and associated cancers of the head and neck and cervix. Int J Cancer 2014;134(3):497-507. doi: 10.1002/ijc.28201. 